\title{
Toward a Unified Scaling Relation for Interfacial Fluxes
}

\author{
Andreas Lorke and Frank Peeters \\ Environmental Physics/Limnological Institute, University of Konstanz, Konstanz, Germany
}

(Manuscript received 22 July 2004, in final form 18 July 2005)

\begin{abstract}
Interfacial fluxes, that is, gas exchange at the water-atmosphere interface and benthic fluxes at the sediment-water interface, are often parameterized in terms of wind speed or turbulent friction velocity, with numerous empirical relationships obtained from individual experiments. The present study attempts to combine the general outcome of such experiments at both interfaces into a universal scaling relation for the thicknesses of the viscous and diffusive sublayers in terms of the Kolmogorov and Batchelor length scales, respectively. Transfer velocities can then be described in terms of the Schmidt number of the respective tracer and in terms of the turbulence dissipation rate. Applying law-of-the-wall scaling to convert dissipation rates into an appropriate friction velocity estimate results in a mechanistic description of the transfer velocity, which is comparable to common empirical parameterizations. It is hypothesized, however, that the dissipation rate and hence the directly estimated level of turbulence provide a more appropriate variable for the parameterization of interfacial fluxes than wind speed or turbulent friction velocity inferred from law-of-the-wall scaling.
\end{abstract}

\section{Introduction}

The physical transport of mass, such as dissolved gases, and heat across the sediment-water and wateratmosphere interfaces is governed by the interplay of exchange processes within an hierarchal structure of sublayers: the turbulent boundary layer, the viscous sublayer, and the diffusive sublayer.

Within the turbulent boundary layer, the level of turbulence usually increases by several orders of magnitude toward the interface. This turbulence is generated by bottom friction in the bottom boundary layers of the atmosphere and the water column, or by wind shear in the surface layer of lakes or oceans. Typical turbulent boundary layer heights range from several meters to several tens or hundreds of meters in lakes and oceans, respectively, and several hundreds of meters to kilometers in the atmosphere. Under certain conditions these layers can be described by law-of-the-wall theory (assuming a constant stress layer) in terms of a friction

Corresponding author address: Andreas Lorke, Limnological Institute, University of Konstanz, Mainaustr. 252, D-78464 Konstanz, Germany.

E-mail: andreas.lorke@uni-konstanz.de velocity $u_{*}$ and a roughness length $z_{0}$ or an equivalent drag coefficient $C_{D}$, resulting in a reciprocal relationship between the turbulence dissipation rate $\varepsilon$ and the distance from the interface $z$,

$$
\varepsilon(z)=\frac{u_{*}^{3}}{\kappa z},
$$

where $\kappa$ is the von Kármán constant $(\kappa \approx 0.41)$.

Significant deviations from the law-of-the-wall scaling of boundary layer turbulence can occur either under convective conditions (Jonas et al. 2003; OcampoTorres et al. 1994; Schimpf et al. 2002; Shay and Gregg 1986), in the presence of (breaking) surface waves (Anis and Moum 1995; Wüest and Lorke 2003) or under non-steady-state conditions, such as in oscillating boundary layers (Lorke et al. 2002; Mellor 2002).

While approaching the interface at scales at which viscous forces are important, turbulent motions become increasingly damped and a viscous sublayer develops with molecular viscosity $\nu$ as the dominating momentum transport mechanism. Viscous sublayers at the seafloor were observed by Chriss and Caldwell (1984), who found the following scaling relation for the height of the viscous sublayer $\delta_{v}$ in terms of the friction velocity from the turbulent boundary layer above: 


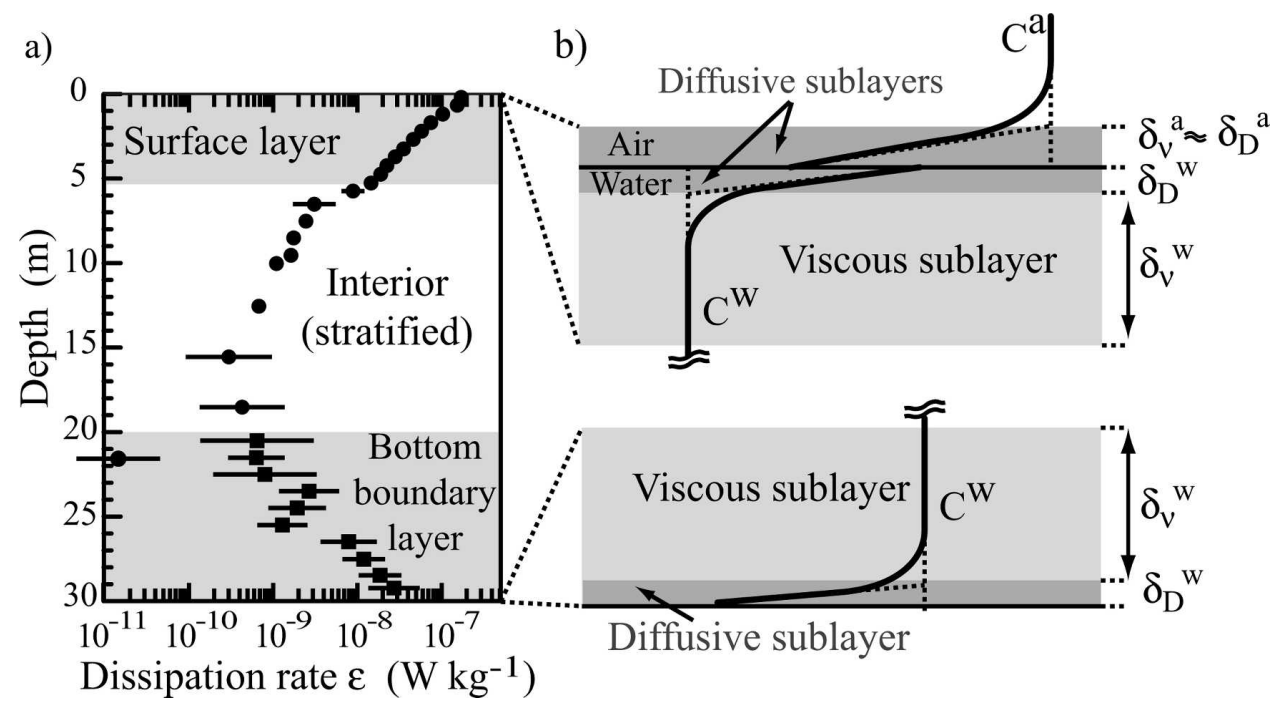

FIG. 1. (a) A typical profile of the turbulence dissipation rate $\varepsilon$ measured in a lake, showing the strong increase of turbulence within the surface and bottom boundary layers (adopted from Wüest and Lorke 2003). (b) Tracer concentration profiles are depicted at the air-water and sediment-water interface schematically for a tracer with a Schmidt number in air of $\mathrm{Sc} \leq 1$ (such as $\mathrm{O}_{2}$ or $\mathrm{CO}_{2}$ ): $\mathrm{C}^{\mathrm{a}}$ and $\mathrm{C}^{\mathrm{W}}$ refer to the tracer concentrations in air and in water, respectively, showing well-mixed distributions within the viscous sublayers of heights $\delta_{v}^{\mathrm{a}}, \delta_{v}^{\mathrm{w}}$, and linear concentration gradients within the diffusive sublayers with the heights $\delta_{\mathrm{D}}^{\mathrm{a}}, \delta_{\mathrm{D}}^{\mathrm{w}}$. For $\mathrm{Sc}=1$ (air side), the viscous and the diffusive sublayers are of equal heights. The concentration discontinuity across the air-water interface reflects solubility considerations.

$$
\delta_{\nu} \simeq 11 \frac{\nu}{u_{*}}
$$

Although turbulent diffusion is damped within the viscous sublayer, the rate of strain of scalar tracer concentration fields creates enhanced concentration gradients, which increase transport by molecular diffusion. Thus, mixing rates of scalar tracers are still enhanced in the viscous sublayer and measured concentration profiles are usually well mixed up to a certain distance from the interface, where molecular diffusion overcomes the regeneration of concentration gradients by viscous straining. At this point, a diffusive sublayer develops with a linear concentration gradient up to the actual interface and the sublayer thickness controls the overall fluxes of mass or heat. The general structure of the boundary layers, including the viscous and diffusive sublayers, is depicted in Fig. 1.

According to the outline above, the height of the diffusive boundary layer $\delta_{D}$ should depend not only on the level of turbulence in the turbulent boundary layer but also on the viscosity of the medium (air or water) and the molecular diffusivity $D$ of the respective tracer. The dependence of $\delta_{D}$ on turbulence can be described, for example, by $u_{*}$, while the dependence of $\delta_{D}$ on viscosity and molecular diffusivity can be expressed by the Schmidt number $\mathrm{Sc}(\mathrm{Sc}=\nu / D)$. Direct measure- ments of diffusive sublayers at the sediment-water interface revealed a relationship of the form

$$
\delta_{D} \simeq \delta_{\nu} \mathrm{Sc}^{n},
$$

(Hondzo 1998) with the Schmidt number exponents $n$ between $1 / 2$ and $1 / 3$.

The diffusive sublayer forms the major bottleneck for the transport of mass or heat, and the respective flux $J$ can be estimated by Fick's law,

$$
J=\frac{D}{\delta_{D}}\left(C_{\delta D}-C_{0}\right)=k\left(C_{\delta D}-C_{0}\right),
$$

with $C_{\delta D}$ and $C_{0}$ denoting the concentrations at the top of the diffusive sublayer and at the interface, respectively, and $k$ denoting the mass transfer coefficient or the transfer velocity. Assuming that the concentration is constant within the viscous sublayer and within the turbulent boundary layer, $C_{\delta D}$ corresponds to the concentration measured in the far-field $C_{\infty}$.

The entire boundary layer structure is mirrored at the air-water interface, and diffusive sublayers exist at the air side as well as at the water side of the interface: the respective Schmidt numbers, the level of turbulence (e.g., $u_{*}$ ), and the associated layer and sublayer heights may differ considerably on the two sides. The overall flux across the air-water interface can be calculated by 


$$
\frac{1}{k_{\mathrm{tot}}}=\frac{1}{K k_{\mathrm{air}}}+\frac{1}{k_{\mathrm{water}}},
$$

where $K$ is the dimensionless Henry coefficient. The mass transfer coefficients $k$ have to be calculated based on the respective position on the air and water side. Depending on the Henry coefficient and the Schmidt number of the scalar tracer under consideration, however, the overall flux is predominately determined by one side in most cases (Jähne and Haußecker 1998).

Much effort has been spent during the last decades in order to describe $k$ as a function of Sc and $u_{*}$, and the general outcome of laboratory and field experiments in lakes and oceans using different measurement techniques for a variety of different tracers (e.g., dissolved gases and heat) converges toward the following relationship:

$$
k=\beta u_{*} \mathrm{Sc}^{n}
$$

(Boudreau 2001; Donelan and Wanninkhof 2002; Jähne et al. 1987; Wanninkhof 1992; Wu 1996). Interestingly, this empirically obtained relationship holds for air-sideand for water-side-controlled surface fluxes as well as for sediment-water fluxes. Experimentally estimated parameters $\beta$ and $n$ in Eq. (6) were usually obtained from individual studies and, hence, for a rather limited range of Schmidt numbers and friction velocities. Estimates obtained from different experiments cover a wide range of variance [see, e.g., Frost and UpstillGoddard (1999) for a comprehensive review].

A number of mechanistic models for air-sea gas exchange were developed [see Frost and Upstill-Goddard (1999) for a review], one of the most successful of which is the boundary layer model by Deacon (1977), with subsequent modifications. Based on the turbulent boundary layer description at a smooth wall of Reichardt (1951), Deacon (1977) derived the following expression for the gas transfer velocity:

$$
k=0.082 u_{*} \mathrm{Sc}^{-2 / 3} .
$$

Observations, however, suggest that the Schmidt number exponent changes to $-1 / 2$ for wind speeds greater than $\sim 5 \mathrm{~m} \mathrm{~s}^{-1}$ (Jähne et al. 1987). It is interesting to note that Dade (1993) derived exactly the same expression as Eq. (7) (with a factor of 0.1 instead of 0.082) for sediment-water transfer velocities.

The friction velocity $u_{*}$, however, can only be an appropriate parameter for estimating interfacial fluxes under the idealized condition of a local equilibrium between the production and dissipation of turbulent kinetic energy in boundary-layer shear flow. Non-steadystate conditions or the additional production of turbu- lent kinetic energy by convective motions or wave breaking are disregarded. Therefore, attempts have been made to describe the gas transfer velocity in terms of the dissipation rate of turbulent kinetic energy directly (e.g., Kitaigorodskii 1984; Lamont and Scott 1970; MacIntyre et al. 1995). The eddy cell model of gas transfer at the air-sea interface presented by Lamont and Scott (1970) results in a scaling relation of the form

$$
k \sim \mathrm{Sc}^{-1 / 2}(\varepsilon \nu)^{1 / 4} .
$$

Several laboratory (Asher and Pankow 1986; Dickey et al. 1984) and field (Zappa et al. 2003) measurements of near-surface turbulence and gas transfer velocities have been shown to agree well with this relationship. Kitaigorodskii and Donelan (1984) proposed a scaling of the gas transfer velocity at the water surface with the Kolmogorov microscales for eddy size and turbulent velocity, and Fairall et al. (2000) used Monin-Obukhov equilibrium scaling to estimate gas fluxes through the viscous and diffusive sublayers.

In this paper we will show that Eq. (8) can be obtained alternatively by very simple scaling arguments. As mentioned by Melville (1996), the thickness of the viscous and diffusive sublayers can be related to the Kolmogorov and Batchelor microscales, respectively, allowing for a derivation of Eq. (8) purely on dimensional arguments.

The principal intention of this paper is not to establish a new model for air-water or sediment-water mass and heat transfer, but rather to broaden the discussion of individual experiments and results toward a mechanistic understanding of interfacial fluxes under the incorporation of some new ideas about the scaling of viscous and diffusive sublayers with boundary layer turbulence, as outlined below.

\section{Toward a unified scaling relation for interfacial fluxes}

Lorke et al. (2003) presented combined measurements of boundary layer turbulence and diffusive sublayer heights for oxygen in the slowly oscillating bottom boundary layer of a lake. Owing to the periodic forcing, the part of the turbulent boundary layer that followed law-of-the-wall scaling was confined to a thin layer above the sediment (Lorke et al. 2002; Mellor 2002). The classical scaling relation according to Eqs. (6) or (7) was not applicable because boundary layer turbulence driven by oscillating currents is poorly defined by $u_{*}$. Similarly, $u_{*}$ is not an appropriate parameter for the scaling of turbulence in studies concerned with airwater gas exchange under convective conditions (Eugster et al. 2003; Schladow et al. 2002) or under the 
influence of wave-enhanced shear (e.g., Bock et al. 1999).

As an alternative approach, Lorke et al. (2003) demonstrated that the scaling of the diffusive sublayer height with the Batchelor length scale for oxygen provided an adequate description of the sediment-water exchange of oxygen observed in the field. The Batchelor length scale $L_{B}$ is defined by

$$
L_{B}=2 \pi\left(\frac{\nu D^{2}}{\varepsilon}\right)^{1 / 4}
$$

and corresponds to the Batchelor wavenumber (Batchelor 1959; Tennekes and Lumley 1973), which describes the microscale of a passive scalar tracer in homogeneous turbulence. In analogy to the Kolmogorov microscale, $L_{B}$ provides the smallest length scales of turbulent tracer fluctuations.

If the proposed scaling $\delta_{D} \simeq L_{B}$ was correct, the same argument could be applied to the viscous sublayer, whose height $\delta_{v}$ should scale with the Kolmogorov length $L_{K}$ :

$$
L_{K}=2 \pi\left(\frac{\nu^{3}}{\varepsilon}\right)^{1 / 4} .
$$

Indeed, evaluating the law of the wall at a height $z=$ $L_{K}$ by substituting Eq. (1) into Eq. (10) results in

$$
L_{K}=(2 \pi)^{4 / 3} \kappa^{1 / 3} \frac{\nu}{u_{*}} \simeq 8.6 \frac{\nu}{u_{*}}
$$

(Lorke et al. 2003), which agrees with the empirical relation from Eq. (2) within the errors given by Chriss and Caldwell (1984). It should be noted that for tracers with Schmidt numbers $\mathrm{Sc} \leq 1$ the Batchelor length scale $L_{B}$ according to Eq. (9) is replaced by the respective formula for the Kolmogorov length scale Eq. (10) (Batchelor 1959).

Taking these two close agreements between observations and estimations from the scaling arguments as evidence for a general scaling relation for interfacial fluxes of the form $\delta_{D} \simeq L_{B}$, the associated transfer velocity $k=D / \delta_{D}$ can be estimated as

$$
k=\frac{1}{2 \pi}\left(\frac{D^{2}}{\nu} \varepsilon\right)^{1 / 4}=\frac{1}{2 \pi} \mathrm{Sc}^{-1 / 2}(\varepsilon \nu)^{1 / 4} .
$$

The dissipation rate estimate, however, is not trivial, since $\varepsilon$ is strongly increasing toward the interface according to Eq. (1), and the relevant estimate should be taken at the top of the viscous sublayer, that is, at a distance $z=L_{K}$ from the interface (viscous dissipation within the viscous sublayer can be regarded as constant). Layer thicknesses of the viscous and diffusive boundary layers for heat and carbon dioxide in air and water, respectively, using typical dissipation rates were estimated, as shown in Table 1.

The above result is equivalent to the scaling relation given in Eq. (8), which was derived by Lamont and Scott (1970) by assuming a sinusoidal current field with localized regions of up- and downwelling near a free fluid surface. However, according to Lamont and Scott, the Schmidt number exponent in Eq. (8) changes from $-1 / 2$ to $-2 / 3$ if a rigid interface is considered. Experimental observations of gas exchange at the water surface for low friction velocities or in the presence of surface films also suggest Schmidt number exponents smaller than $-1 / 2$ (e.g., Jähne et al. 1987). The Batchelor length scale concept described above [Eq. (12)] cannot predict a change in the Schmidt number dependence, suggesting that the assumptions of the model are not valid if, for example, turbulence levels are low. The Batchelor length scale [Eq. (9)] was derived for conditions of homogeneous and isotropic turbulence, and Eq. (12) additionally assumes steady-state conditions in the diffusive sublayer. If friction velocities are low (low wind speeds) and temporal averaging intervals are long, the intermittent nature of turbulence becomes increasingly important. Intermittent turbulence is characterized by a motion that is "sometimes turbulent and sometimes non-turbulent" (Seuront et al. 2005). During the adjustment to a new steady state and especially during the nonturbulent time periods, the interfacial exchange depends more strongly on molecular diffusion, resulting in a decreasing Schmidt number exponent. Turbulence in the vicinity of an interface cannot be regarded as isotropic either since a considerable range of the dissipation wavenumbers is suppressed in the vertical direction (Doron et al. 2001). The effect of anisotropy becomes increasingly important with increasing turbulent length scales and it can be expected to be more important near a rigid interface than at a free surface. Thus, the increasing thickness of the viscous and diffusive sublayers with decreasing dissipation

TABLE 1. Typical layer thicknesses of the viscous sublayer $\delta_{v}$ and the diffusive sublayer $\delta_{D}$ for heat and carbon dioxide in water and air; $\delta_{\nu}$ and $\delta_{D}$ were calculated using Eqs. (9) and (8), respec-

\begin{tabular}{|c|c|c|c|c|}
\hline & $\begin{array}{l}\text { Heat in } \\
\text { water }\end{array}$ & $\begin{array}{c}\text { Heat in } \\
\text { air }\end{array}$ & $\begin{array}{l}\mathrm{CO}_{2} \text { in } \\
\text { water }\end{array}$ & $\begin{array}{c}\mathrm{CO}_{2} \text { in } \\
\text { air }\end{array}$ \\
\hline $\mathrm{Sc}$ & 10 & 0.6 & 600 & 1 \\
\hline$\delta_{\nu}=L_{K}(\mathrm{~m})$ & $1.1 \times 10^{-2}$ & $6.5 \times 10^{-3}$ & $1.1 \times 10^{-2}$ & $6.5 \times 10^{-3}$ \\
\hline$\delta_{D}=L_{B}(\mathrm{~m})$ & $3.5 \times 10^{-3}$ & $8 \times 10^{-3}$ & $4.5 \times 10^{-4}$ & $6.5 \times 10^{-3}$ \\
\hline
\end{tabular}
tively, using a dissipation rate of $1 \times 10^{-7} \mathrm{~W} \mathrm{~m}^{-2}$ in water and $3 \times 10^{-3} \mathrm{~W} \mathrm{~m}^{-2}$ in the atmosphere. The respective Schmidt numbers Sc (corresponding to a temperature of $20^{\circ} \mathrm{C}$ ) are shown. 


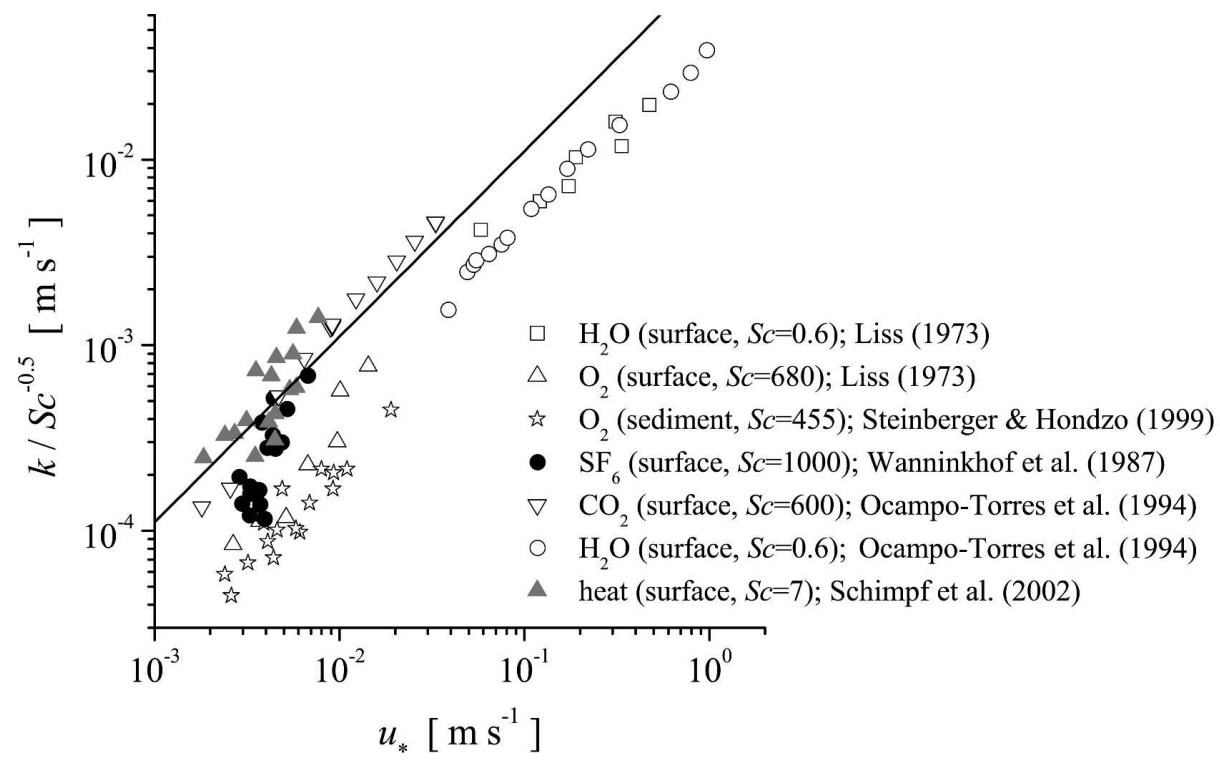

FIG. 2. Gas transfer velocity $k$, normalized by the square root of the Schmidt number Sc, vs friction velocity $u_{*}$ from a variety of field (filled symbols) and laboratory (open symbols) measurements. The kind of tracer, the respective Schmidt number, and the data source are given in the legend. Note that $u_{*}$ refers to the friction velocity in the atmospheric boundary layer for air-side-controlled surface fluxes and to the friction velocity in water for water-side-controlled fluxes (the same applies for Sc). The data were plotted as published originally, with no additional corrections for the temperature dependence of Sc or for varying drag coefficients $C_{D}$. The solid line shows the parameterization from Eq. (13).

rates (e.g., due to decreasing wind speeds at the water surface) leads to a growing suppression of turbulent transport in the vertical and to an increasing importance of molecular diffusion. Though only speculative, intermittency and anisotropy of turbulence can lead to the observed increase of the Schmidt number exponent at low levels of turbulence.

\section{Law-of-the-wall conditions}

Under conditions where the law of the wall applies, Eq. (12) can easily be compared with other parameterizations and with measurements that usually do not refer to $\varepsilon$ but rather to $u_{*}$ or the wind speed, respectively. Substituting Eq. (1) into Eq. (12) yields

$$
k=\frac{1}{(2 \pi)^{4 / 3} \kappa^{1 / 3}} \mathrm{Sc}^{-1 / 2} u_{*} \simeq \frac{1}{9} \mathrm{Sc}^{-1 / 2} u_{*} .
$$

This expression agrees with the general outcome of many experimental observations and conceptual models [Eq. (6); Frost and Upstill-Goddard 1999]. The estimated transfer velocity is about a factor of 2 higher than the one obtained from an empirical parameterization for a rough surface $\left[k=(1 / 16) \mathrm{Sc}^{-1 / 2} u_{*} ;\right.$ Jähne et al. 1987]. It should be noted that Eq. (13) is valid for the atmosphere (air-side-controlled surface fluxes, e.g., wa- ter vapor or heat) as well as for the water column (water-side-controlled surface fluxes and sediment-water fluxes, e.g., $\mathrm{CO}_{2}, \mathrm{O}_{2}$, or $\mathrm{CH}_{4}$ ). The values of $u_{*}$ and $\mathrm{Sc}$ have to be chosen according to the location of the respective boundary layer, that is, for air or water. Equation (5) can be used for fluxes controlled by air-side and water-side transport.

The parameterization in Eq. (13) is compared with experimental observations in Fig. 2. The datasets were chosen according to their availability from publications and represent air-side- and water-side-controlled surface fluxes as well as sediment-water fluxes measured in the laboratory, in lakes, or in the ocean. Although the scatter between individual experiments and the deviations from the parameterization from Eq. (13) are large, the general trend of the experimental data is reproduced and no general differences between air-sideand water-side-controlled fluxes or surface and sediment-water fluxes can be deduced. There is rather a tendency that field observations are better reproduced by Eq. (13) than laboratory estimates, which may be related to the particular choice of datasets, however.

It should be noted that Eq. (13) requires the validity of the law of the wall [Eq. (1)] and is therefore only a special case of the scaling relation proposed in Eq. (12). The law of the wall does not generally apply, and it is specifically questionable in surface waters affected by 
wave action and convection. Unfortunately, no direct dissipation measurements are available for published transfer velocities, and almost all measurements rely on an estimation of $u_{*}$ from wind measurements at some level above the water surface or on special scaling relations established for the particular laboratory facility (e.g., Jähne et al. 1987; Ocampo-Torres et al. 1994). Moreover, almost all studies used a constant surface drag coefficient $C_{D}$ in order to estimate $u_{*}$ from measured wind speeds [the drag coefficient is related to the roughness length $z_{0}$ by $\left.C_{D}^{2}=\kappa / \ln \left(1 \mathrm{~m} / z_{0}\right)\right]$. A review by Wüest and Lorke (2003), however, revealed that $C_{D}$ has a minimum at a wind speed of $\sim 5 \mathrm{~m} \mathrm{~s}^{-1}$. Below 5 $\mathrm{m} \mathrm{s}^{-1} C_{D}$ increases strongly with decreasing wind speed and above $5 \mathrm{~m} \mathrm{~s}^{-1} C_{D}$ increases moderately with increasing wind speed. The dependence of $C_{D}$ on wind speed could not only explain the large scatter within and among the different datasets, but also the frequently observed increase of the transfer velocity at wind speeds exceeding $\sim 5 \mathrm{~m} \mathrm{~s}^{-1}$ (Deacon 1977; Jähne et al. 1987) and its strong correlation with surface roughness or the mean square slope of short wind waves (Bock et al. 1999; Jähne et al. 1987).

\section{Conclusions and outlook}

Interfacial fluxes at the air-water and sediment water interfaces can be described jointly by using relatively simple scaling relations. These scaling relations are based on the microscales of turbulence and turbulent mixing and on the assumption of a structured layering with a diffusive boundary layer in the vicinity of the interface limiting transport. The resulting dependence of the estimated transfer velocities on the Schmidt number and the turbulent friction velocity is comparable to common empirical parameterizations and experimental observations. The major difference, however, is that the interfacial fluxes are described in terms of the turbulence dissipation rate instead of wind speed or friction velocity. This approach enables an estimation of transfer velocities in situations where boundary layer turbulence cannot be described by wall-layer scaling, for example, for convective mixing and for oscillating currents. It could be hypothesized that this represents a more direct approach for estimating transfer velocities under natural conditions since it decouples the parameterization of the momentum transfer from the actual mass transfer and thus eliminates uncertainties, for example, associated with the estimation of a representative roughness length or drag coefficient.

Although the simplification in parameterization achieved by describing the surface gas transfer in terms of the wind speed appears to be practical for model applications, it does not result in a deterministic understanding of the processes involved and may result in erroneous model results under convective conditions or for oscillating flow regimes.

The large scatter in experimental observations of transfer velocities might be, at least partially, explained by the usage of a scaling relation based on $u_{*}$ rather than on $\varepsilon$. Hence, there is a need for a new generation of experiments, combining direct boundary layer turbulence measurements with estimations of mass transfer velocities. New measurement techniques capable of resolving transfer velocities on small spatial scales and short time scales, such as the controlled flux method (Jähne et al. 1989; Schimpf et al. 2002), are a promising tool for such experiments.

Acknowledgments. We thank J. C. Little, D. F. McGinnis, and two anonymous reviewers for providing constructive comments on early versions of this paper. The work was financially supported by the German Research Foundation within the framework of the Collaborative Research Center 454: The littoral zone of Lake Constance.

\section{REFERENCES}

Anis, A., and J. N. Moum, 1995: Surface wave-turbulence interactions: Scaling $\varepsilon(z)$ near the sea surface. J. Phys. Oceanogr., 25, 2025-2045.

Asher, W. E., and J. F. Pankow, 1986: The interaction of mechanically generated turbulence and interfacial films with a liquid phase controlled gas/liquid transport process. Tellus, 38B, 305-318.

Batchelor, G. K., 1959: Small-scale variation of convected quantities like temperature in turbulent fluid. J. Fluid Mech., 5, 113-133.

Bock, E. J., T. Hara, N. M. Frew, and W. R. McGillis, 1999: Relationship between air-sea gas transfer and short wind waves. J. Geophys. Res., 104, 25 821-25 831.

Boudreau, B. P., 2001: Solute transport above the sediment-water interface. The Benthic Boundary Layer, B. P. Boudreau and B. B. Jørgensen, Eds., Oxford University Press, 104-143.

Chriss, T. M., and D. R. Caldwell, 1984: Universal similarity and the thickness of the viscous sublayer at the ocean floor. $J$. Geophys. Res., 89, 6403-6414.

Dade, W. B., 1993: Near-bed turbulence and hydrodynamic control of diffusional mass transfer at the sea floor. Limnol. Oceanogr., 38, 52-69.

Deacon, E. L., 1977: Gas transfer to and across an air-water interface. Tellus, 29, 363-374.

Dickey, T. D., B. Hartman, D. Hammond, and E. Hurst, 1984: A laboratory technique for investigating the relationship between gas transfer and fluid turbulence. Gas Transfer at $\mathrm{Wa}$ ter Surfaces, W. Brutsaert and G. Jirka, Eds., D. Reidel, 93100.

Donelan, M. A., and R. Wanninkhof, 2002: Gas transfer at water surfaces-Concepts and issues. Gas Transfer at Water Surfaces, M. A. Donelan et al., Eds., Amer. Geophys. Union, 1-10.

Doron, P., L. Bertuccioli, J. Katz, and T. Osborn, 2001: Turbu- 
lence characteristics and dissipation estimates in the coastal ocean bottom boundary layer using PIV data. J. Phys. Oceanogr., 31, 2108-2134.

Eugster, W., G. Kling, T. Jonas, J. P. McFadden, A. Wüest, S. MacIntyre, and F. S. Chapin III, 2003: $\mathrm{CO}_{2}$ exchange between air and water in an Arctic Alaskan and midlatitude Swiss lake: Importance of convective mixing. J. Geophys. Res., 108, 4362, doi:10.1029/2002JD002653.

Fairall, C. W., J. E. Hare, J. B. Edson, and W. R. McGillis, 2000: Parameterization and micrometeorological measurement of air-sea gas transfer. Bound.-Layer Meteor., 96, 63-105.

Frost, T., and R. C. Upstill-Goddard, 1999: Air-sea gas exchange into the millennium: Progress and uncertainties. Oceanogr. Mar. Biol., 37, 1-45.

Hondzo, M., 1998: Dissolved oxygen transfer at the sedimentwater interface in a turbulent flow. Water Resour. Res., 34, 3525-3533.

Jähne, B., and H. Haußecker, 1998: Air-water gas exchange. Annu. Rev. Fluid Mech., 30, 443-468.

— - K. O. Münnich, R. Bösinger, A. Dutzi, W. Huber, and P. Libner, 1987: On the parameters influencing air-water gas exchange. J. Geophys. Res., 92, 1937-1949.

— , P. Libner, R. Fischer, T. Billen, and E. J. Plate, 1989: Investigating the transfer processes at the aqueous viscous boundary layer by the controlled flux method. Tellus, 41B, 177-195.

Jonas, T., A. Stips, W. Eugster, and A. Wüest, 2003: Observations of a quasi shear-free lacustrine convective boundary layer: Stratification and its implications on turbulence. J. Geophys. Res., 108, 3328, doi:10.1029/2002JC001440.

Kitaigorodskii, S. A., 1984: On the fluid dynamical theory of turbulent gas transfer across an air-sea interface in the presence of breaking wind-waves. J. Phys. Oceanogr., 14, 960-972.

— fer. Gas Transfer at Water Surfaces, W. Brutsaert and G. Jirka, Eds., D. Reidel, 147-170.

Lamont, J. C., and D. S. Scott, 1970: An eddy cell model of mass transfer into the surface of a turbulent liquid. AIChE J., 16, 513-519.

Liss, P. S., 1973: Processes of gas exchange across an air-water interface. Deep-Sea Res., 20, 221-238.

Lorke, A., L. Umlauf, T. Jonas, and A. Wüest, 2002: Dynamics of turbulence in low-speed oscillating bottom-boundary layers of stratified basins. Environ. Fluid Mech., 2, 291-313.

, B. Müller, M. Maerki, and A. Wüest, 2003: Breathing sediments: The control of diffusive transport across the sedimentwater interface by periodic boundary-layer turbulence. Limnol. Oceanogr., 48, 2077-2085.
MacIntyre, S., R. Wanninkhof, and J. P. Chanton, 1995: Trace gas exchange across the air-water interface in freshwater and coastal marine environments. Biogenic Trace Gases: Measuring Emissions from Soil and Water, P. A. Matson and R. C. Harris, Eds., Blackwell Science, 52-97.

Mellor, G. L., 2002: Oscillatory bottom boundary layers. J. Phys. Oceanogr., 32, 3075-3088.

Melville, W. K., 1996: The role of surface-wave breaking in airsea interaction. Annu. Rev. Fluid Mech., 28, 279-321.

Ocampo-Torres, F. J., M. A. Donelan, N. Merzi, and F. Jia, 1994: Laboratory measurements of mass transfer of carbon dioxide and water vapour for smooth and rough flow conditions. Tellus, 46B, 16-32.

Reichardt, H., 1951: Vollständige Darstellung der turbulenten Geschwindigkeitsverteilung in glatten Leitungen. Z. Angew. Math. Mech., 31, 208-219.

Schimpf, U., B. Jähne, and H. Haußecker, 2002: On the investigations of statistical properties of the micro turbulence at the ocean surface. Gas Transfer at Water Surfaces, 1st ed. M. A. Donelan et al., Eds., Amer. Geophys. Union, 51-57.

Schladow, G., L. Lee, B. E. Hürzeler, and P. B. Kelly, 2002: Oxygen transfer across the air-water interface by natural convection in lakes. Limnol. Oceanogr., 47, 1394-1404.

Seuront, L., H. Yamazaki, and F. G. Schmitt, 2005: Intermittency. Marine Turbulence, H. Baumert, J. H. Simpson, and J. Sündermann, Eds., Cambridge University Press, 66-78.

Shay, T. J., and M. C. Gregg, 1986: Convectively driven mixing in the upper ocean. J. Phys. Oceanogr., 16, 1777-1799.

Steinberger, N., and M. Hondzo, 1999: Diffusional mass transfer at sediment-water interface. J. Environ. Eng., 125, 192-200.

Tennekes, H., and J. L. Lumley, 1973: A First Course in Turbulence. MIT Press, 300 pp.

Wanninkhof, R., 1992: Relationship between wind speed and gas exchange over the ocean. J. Geophys. Res., 97, 7373-7382.

- J. R. Ledwell, and W. S. Broecker, 1987: Gas exchange on Mono Lake and Crowley Lake, California. J. Geophys. Res., 92, 14 567-14 580 .

Wu, J., 1996: Air-sea gas transfer: Mechanisms and parameterization. J. Phys. Oceanogr., 26, 1440-1447.

Wüest, A., and A. Lorke, 2003: Small-scale hydrodynamics in lakes. Annu. Rev. Fluid Mech., 35, 373-412.

Zappa, C. J., P. A. Raymond, E. A. Terray, and W. R. McGillis, 2003: Variation in surface turbulence and the gas transfer velocity over a tidal cycle in a macro-tidal estuary. Estuaries, 26, 1401-1415. 